\title{
Prevalência de sobrepeso e obesidade em nipo-brasileiros: comparação entre sexos e geração ${ }^{1}$
}

\author{
Prevalence of overweight and obesity among \\ Japanese-Brazilian: comparison across \\ sex and generation
}

Rosana Farah SIMONY2,3

Suely Godoy Agostinho GIMENO 4

Sandra Roberta Gouvea FERREIRA ${ }^{4}$

Laércio Joel FRANCO 5

Japanese-Brazilian Diabetes Study Group ${ }^{6}$

\section{RE S U M O}

\section{Objetivo}

Descrever a prevalência de sobrepeso, obesidade e obesidade abdominal em nipo-brasileiros residentes na cidade de Bauru (SP), Brasil.

\section{Métodos}

Os dados foram obtidos a partir de um estudo transversal com 1330 nipo-brasileiros de $1^{\text {a }}$ e $2^{\text {a }}$ geração, de ambos os sexos, com idade $\geq 30$ anos. Os critérios para sobrepeso e obesidade foram índice de massa corporal entre $25-29,9 \mathrm{~kg} / \mathrm{m}^{2}$ e $\geq 30 \mathrm{~kg} / \mathrm{m}^{2}$, respectivamente. A obesidade abdominal foi classificada com valores de circunferência da cintura $\geq 90 \mathrm{~cm}$, para homens, e $\geq 80 \mathrm{~cm}$ para mulheres. Foram calculadas as prevalências de sobrepeso, obesidade e obesidade abdominal por ponto e por intervalo, com 95\% de confiança.

\section{Resultados}

Verificou-se que a prevalência de sobrepeso em nipo-brasileiros foi de $26,1 \%$ e $27,9 \%$ na primeira geração e de $44,8 \%$ e $32,5 \%$ na segunda geração, respectivamente, para homens e mulheres. Em relação à obesidade,

\footnotetext{
1 Apoio: Fundação de Amparo à Pesquisa do Estado de São Paulo (processo 00/01162-4).

2 Centro Universitário São Camilo, Curso de Nutrição. Av. Nazaré, 1501, Ipiranga, 05025-110, São Paulo, SP, Brasil.

Correspondência para/Correspondence to: R.F. SIMONY. E-mail: <rosanafarah@terra.com.br>.

${ }^{3}$ Universidade Presbiteriana MacKenzie, Curso de Nutrição. São Paulo, SP, Brasil.

${ }^{4}$ Universidade de São Paulo, Faculdade de Saúde Pública, Departamento de Nutrição. São Paulo, SP, Brasil.

${ }^{5}$ Universidade de São Paulo, Faculdade de Medicina de Ribeirão Preto, Departamento de Medicina. Ribeirão Preto, SP, Brasil.

${ }^{6}$ Japanese-Brazilian, Diabetes Study Group. São Paulo, SP, Brasil.
} 
170 | R.F. SIMONY et al.

a prevalência entre homens foi de 3,7\% e 12\%, e nas mulheres de 6,6\% e 9,9\% respectivamente na primeira e na segunda geração. Observou-se aumento na prevalência de sobrepeso e obesidade nos homens entre as gerações, apesar de as diferenças não serem estatisticamente significantes. A obesidade abdominal nos homens de primeira e segunda geração foi de 32,1\% e 45,3\%, e nas mulheres estes valores foram de 49,2\% e $48,5 \%$, respectivamente. No período de estudo não foram observados aumentos nas prevalências de sobrepeso e obesidade estatisticamente significantes $(p<0,05)$.

\section{Conclusão}

O aumento percentual na prevalência de sobrepeso e obesidade abdominal em nipo-brasileiros pode ser, em parte, explicado pelo processo da ocidentalização, reforçando a necessidade de medidas preventivas, visando a minimizar as conseqüências metabólicas da obesidade nos nipo-brasileiros.

Termos de indexação: Circunferência abdominal. Índice de Massa Corporal. Migrantes. Obesidade. Sobrepeso.

\section{A B S T R A C T}

\section{Objective}

To describe the prevalence of overweight, obesity and abdominal obesity in Japanese-Brazilians living in Bauru city, São Paulo State, Brazil.

\section{Methods}

Data were from a cross-sectional population-based study of 1.330 Japanese-Brazilians from the first and second generation of both genders and aged $\geq 30$ years. Overweight and obesity criteria were body mass index of $25-29.9 \mathrm{~kg} / \mathrm{m}^{2}$ and $\geq 30 \mathrm{~kg} / \mathrm{m}^{2}$, respectively. Abdominal obesity was classified by waist circumference $\geq 90 \mathrm{~cm}$ for men and $\geq 80 \mathrm{~cm}$ for women. Point and 95\%-confidence-interval prevalences were calculated for overweight, obesity and abdominal obesity.

\section{Results}

In the first generation of Japanese-Brazilians, $26.1 \%$ of men and $27.9 \%$ of women were overweight in comparison with $44.8 \%$ e $32.5 \%$ in the second generation for males and females, respectively. The prevalence of obesity was $3.7 \%$ and $12.0 \%$ in males and $6.6 \%$ and $9.9 \%$ in females respectively for the first and second generation. Overweight and obesity rates for men increased between generations, even though the differences were not statistically significant. Abdominal obesity in first and second-generation men was 32.1\% and 45.3 $\%$ respectively and among women these rates were $49.2 \%$ and $48.5 \%$, respectively. The increased prevalence of overweight and abdominal obesity in the study period was not statistically significant $(p<0.05)$.

\section{Conclusion}

The high rates of overweight and central obesity among the Japanese-Brazilians immigrants can be partly explained by the westernization process. There is a strong need for preventive measures that minimize the metabolic consequences of excess weight in Japanese-Brazilians.

Indexing terms: Abdominal circumference. Body mass index. Transients and migrants. Obesity. Overweight.

\section{N T R O D U ÇÃ O}

Nas últimas décadas foi observado um aumento significativo na prevalência da obesidade em todo o mundo ${ }^{1-5}$, sendo a mesma atualmente considerada um importante problema de saúde pública em muitos países, por se encontrar associada a doenças crônicas como a síndrome metabólica, a hipertensão e o diabetes mellitus $(\mathrm{DM} 2)^{6}$. Segundo Popkin7, a obesidade tornou-se um problema de saúde mundial e por isso é considerada uma epidemia global, sendo a principal causa de muitas doenças tanto em países desenvolvidos como naqueles em desenvolvimento.

Segundo dados da Pesquisa Nacional de Nutrição do Japão, as prevalências de sobrepeso e obesidade, no sexo masculino, foram de $24,5 \%$ e $2,3 \%$ e $17,8 \%$ e $3,4 \%$ entre mulheres acima dos 20 anos de idade ${ }^{8}$. Apesar de a população de adultos obesos ser menor do que aquela observada 
em sociedades ocidentais e em outros países asiáticos, a prevalência de excesso de peso (sobrepeso e obesidade) atinge atualmente quase um quarto da população adulta japonesa ${ }^{9}$.

A observação de que populações migrantes apresentam padrões distintos de morbimortalidade, quando comparadas ao perfil dos residentes do seu local de origem, desperta grande interesse científico, pois permite avaliar o efeito do meio ambiente no aparecimento de doenças (mudanças no seu estilo de vida devido ao processo de adaptação ao novo ambiente $)^{10}$.

Um estudo recente conduzido por Yoshiike et al. ${ }^{11}$, mostrou um aumento na prevalência de sobrepeso em homens residentes em áreas rurais do Japão, sugerindo que mudanças no grau de atividade física, assim como na automatização da agricultura, poderiam ser as responsáveis pela mudança neste perfil nutricional. Os autores sugerem, ainda, que essas diferenças entre as áreas rurais e urbanas podem ser, em parte, explicadas por fatores sociais e de estilo de vida que podem influenciar o aumento das prevalências do excesso de peso. Essa hipótese é reforçada por resultados de estudos conduzidos em populações de migrantes de áreas rurais e urbanas, que sugerem que a diminuição na atividade física, associada a mudanças na dieta usual, é responsável pelo aumento na prevalência da obesidade reforçando a hipótese da importância da aculturação sobre a etiologia do sobrepeso ${ }^{11}$.

A população migrante japonesa é um dos exemplos das conseqüências do processo de aculturação. Isso porque, atualmente, apresenta elevada prevalência de DM2 e de dislipidemia, provavelmente, como uma das conseqüências das mudanças da dieta e da atividade física nas últimas décadas ${ }^{12}$.

Primeiramente, os japoneses apresentavam baixas prevalências de DM2, hipertensão e dislipidemia, porém estudos realizados em japoneses imigrantes vivendo nos Estados Unidos mostraram que esse quadro havia sido modificado $^{12-14}$. No Brasil, Souza \& Gotlieb ${ }^{10}$, em um estudo conduzido com nipo-brasileiros no Estado do Paraná, verificaram que houve modificação do padrão de mortalidade de indivíduos de primeira geração, quando comparados aos que permaneceram no seu país de origem (Japão), sendo este semelhante ao perfil do local de destino, sugerindo a influência de fatores socioculturais sob essas diferenças. Ferreira et al. ${ }^{15}$ verificaram que os nipo-brasileiros entre 40 e 79 anos apresentaram elevada prevalência de hipertensão sendo, talvez, resultado da influência desfavorável da ocidentalização no desenvolvimento de doenças crônicas não transmissíveis. Gimeno et al. ${ }^{16}$ verificaram, no período de 1993 a 2000, uma piora no perfil glicêmico dos nipo-brasileiros e elevadas prevalências de intolerância à glicose (DM2, glicemia de jejum alterada e tolerância diminuída à glicose), sendo, atualmente, considerada uma das maiores do mundo.

As evidências apontadas sobre a elevada prevalência de doenças crônicas em japoneses que, em alguns momentos de suas vidas, migraram para ambientes culturalmente distintos, reforça o papel da obesidade neste grupo populacional. Não somente a obesidade geral, mas especialmente a obesidade abdominal (OA) a qual faz parte da história natural da intolerância à glicose e de diversas outras doenças crônicas. Assim sendo, o objetivo deste trabalho foi descrever as prevalências de sobrepeso, obesidade e obesidade abdominal na população de nipo-brasileiros residentes na cidade de Bauru, em 2000.

\section{MÉ TO D OS}

O Brasil tem uma das maiores populações de migrantes do Japão, representando, em 1987, 1288000 indivíduos $(0,9 \%$ da população brasileira). Destes, 828 mil indivíduos (64,3\%) moram no Estado de São Paulo (2,9\% da população do estado). Em Bauru, a colonização japonesa teve início em 1914, principalmente na área rural. Os japoneses, a princípio, vieram para trabalhar nas plantações de café, sendo que atualmente somente $11,0 \%$ destes imigrantes ainda trabalham na área rural. 
Teve início em 1993 a primeira fase do estudo com 647 nipo-brasileiros residentes na cidade de Bauru, Estado de São Paulo, com o objetivo principal de conhecer a prevalência de DM2 e outras doenças associadas neste grupo de migrantes japoneses de primeira (Issei) e segunda geração (Nissei). Definiram-se como Issei todos os indivíduos nascidos no Japão e Nisseis os filhos de Isseis. Todos foram examinados e submetidos ao teste de tolerância oral à glicose.

Em 1999, teve início a segunda fase do estudo na qual, além dos participantes da primeira fase, foram convidados a participar todos os nipobrasileiros de primeira e segunda geração com idade $\geq 30$ anos ( $n=1751$ indivíduos). Detalhes sobre as características, a seleção e o recrutamento desta população já foram descritos ${ }^{17}$. Todos os indivíduos identificados foram convidados a participar do estudo, sendo que 1330 (76,0\%) responderam positivamente ao convite. Entre aqueles que não participaram do estudo, observouse maior proporção de indivíduos do sexo masculino e com idade $\leq 60$ anos, quando comparados aos participantes $(p<0,05)$. Das $421(24,0 \%)$ desistências, 92 (5,3\%) foram por óbitos, 272 $(16,0 \%)$ por recusa e $57(3,3 \%)$ por mudança de cidade ou país.

Após concordarem em participar da pesquisa (protocolo aprovado pelo comitê de ética em pesquisa da UNIFESP, número 082/98) e terem assinado o termo de consentimento, foram agendados local e data onde os indivíduos deveriam comparecer para a realização do exame físico. Durante o exame físico foram tomadas as medidas antropométricas por profissionais treinados, que pesaram e mediram os participantes com roupas leves e sem sapatos. Uma balança digital calibrada, da marca Filizola ${ }^{\circledR}$, e um estadiômetro acoplado à parede foram os instrumentos utilizados para a tomada do peso e da altura, respectivamente. O índice de massa corporal (IMC) foi calculado dividindo-se o peso $(\mathrm{kg})$ pelo quadrado da altura $\left(\mathrm{m}^{2}\right)$. Para a medição da circunferência da cintura (CC) utilizou-se fita métrica, medida em torno da cicatriz umbilical (indivíduos em pé).
O sobrepeso e a obesidade foram definidos de acordo com os pontos de corte recomendados pela Organização Mundial da Saúde ${ }^{18}$, ou seja, IMC 25-29,9kg/m² e IMC $\geq 30,0 \mathrm{~kg} / \mathrm{m}^{2}$, respectivamente, para os sexos. A obesidade abdominal (OA) foi definida como a $C C \geq 80 \mathrm{~cm}$ para mulheres e $\geq 90 \mathrm{~cm}$ para homens, valores recomendados para a população asiática ${ }^{19}$.

As prevalências de sobrepeso, obesidade e obesidade abdominal foram estimadas por ponto e por intervalo com intervalo de confiança (IC) de 95\% para ambas as gerações.

O software utilizado para análise estatística foi o STATA $7.0^{20}$.

\section{RES ULTADOS}

Dos 1330 participantes, 717 (53,9\%) eram do sexo feminino e $613(46,1 \%)$ do sexo masculino. A maior parte dos indivíduos pertencia à segunda geração de migrantes japoneses $(80,7 \%)$ e tinha menos que 60 anos de idade (57,3\%).

A Tabela 1 mostra a porcentagem de nipo-brasileiros com sobrepeso (IMC entre 25 e $\left.29,9 \mathrm{~kg} / \mathrm{m}^{2}\right)$ e obesidade $\left(\mathrm{IMC} \geq 30,0 \mathrm{~kg} / \mathrm{m}^{2}\right)$ e a Tabela 2 mostra a prevalência de OA, ambas, segundo idade e geração. Observa-se que houve um aumento nas razões de prevalência de sobre-

Tabela 1. Nipo-brasileiros com sobrepeso (IMC $\left.\geq 25 \mathrm{~kg} / \mathrm{m}^{2}\right)$, segundo idade e geração. Bauru (SP), 2000.

\begin{tabular}{|c|c|c|c|c|c|c|}
\hline \multirow{3}{*}{$\begin{array}{l}\text { Faixa etária } \\
\text { (anos) }\end{array}$} & \multicolumn{4}{|c|}{$\mathrm{IMC} \geq 25 \mathrm{~kg} / \mathrm{m}^{2}$} & \multirow{2}{*}{\multicolumn{2}{|c|}{ Total }} \\
\hline & \multicolumn{2}{|c|}{ Primeira geração } & \multicolumn{2}{|c|}{ Segunda geração } & & \\
\hline & $\mathrm{n}$ & $\%$ & $n$ & $\%$ & $n$ & $\%$ \\
\hline$<45$ & 1 & 1,22 & 91 & 17,3 & 92 & 15,2 \\
\hline $45-49$ & 5 & 6,10 & 73 & 13,9 & 78 & 12,9 \\
\hline $50-54$ & 8 & 9,80 & 95 & 18,1 & 103 & 16,0 \\
\hline $55-59$ & 7 & 8,50 & 93 & 17,7 & 100 & 16,5 \\
\hline $60-64$ & 6 & 7,30 & 77 & 14,7 & 83 & 13,7 \\
\hline $65-69$ & 14 & 17,10 & 62 & 11,8 & 76 & 12,5 \\
\hline $70-74$ & 22 & 26,80 & 26 & 4,9 & 48 & 7,9 \\
\hline $75-79$ & 14 & 17,10 & 7 & 1,3 & 21 & 3,5 \\
\hline$\geq 80$ & 5 & 6,10 & 1 & 0,2 & 6 & 0,9 \\
\hline Total & 82 & 100,00 & 525 & 100,0 & 607 & 100,0 \\
\hline
\end{tabular}

IMC: índice de massa corporal. 
peso e OA em quase todos os grupos etários, porém este aumento não foi estatisticamente significante $(p>0,05)$.

A Tabela 3 mostra as razões de prevalência de sobrepeso, obesidade e obesidade abdominal segundo sexo e geração. Houve uma pequena porcentagem de indivíduos com peso normal com OA (9,6\% na primeira geração e $7,7 \%$ na segunda geração), o mesmo ocorrendo no caso de indivíduos com sobrepeso sem OA $(17,1 \%)$ entre os homens. Para o sexo feminino, observou-se que somente $26,3 \%$ na primeira geração e 19,7\% na segunda geração tinham peso normal sem $O A$. Para aquelas com sobrepeso apenas $8,8 \%$ e

Tabela 2. Nipo-brasileiros com obesidade abdominal segundo idade e geração. Bauru (SP), 2000.

\begin{tabular}{|c|c|c|c|c|c|c|}
\hline \multirow{3}{*}{$\begin{array}{l}\text { Faixa etária } \\
\text { (anos) }\end{array}$} & \multicolumn{4}{|c|}{ Circunferência da cintura $\geq 90 / \geq 80 \mathrm{~cm}$} & \multirow{2}{*}{\multicolumn{2}{|c|}{ Total }} \\
\hline & \multicolumn{2}{|c|}{ Primeira geração } & \multicolumn{2}{|c|}{ Segunda geração } & & \\
\hline & $n$ & $\%$ & $\mathrm{n}$ & $\%$ & $n$ & $\%$ \\
\hline$<45$ & 1 & 0,9 & 70 & 13,8 & 71 & 11,7 \\
\hline $45-49$ & 7 & 6,8 & 65 & 12,9 & 72 & 11,8 \\
\hline $50-54$ & 6 & 5,8 & 89 & 17,6 & 95 & 15,6 \\
\hline $55-59$ & 6 & 5,8 & 94 & 18,6 & 100 & 16,5 \\
\hline $60-64$ & 8 & 7,8 & 78 & 15,5 & 86 & 14,1 \\
\hline $65-69$ & 18 & 17,5 & 64 & 12,7 & 82 & 13,5 \\
\hline 70-74 & 23 & 22,3 & 33 & 6,5 & 56 & 9,2 \\
\hline 75-79 & 21 & 20,4 & 8 & 1,6 & 29 & 4,8 \\
\hline$\geq 80$ & 13 & 12,6 & 4 & 0,8 & 17 & 2,8 \\
\hline Total & 103 & 100,0 & 505 & 100,0 & 608 & 100,0 \\
\hline
\end{tabular}

$16,1 \%$, respectivamente, da primeira e da segunda geração não tinham OA. Todos os indivíduos com IMC $\geq 30,0 \mathrm{~kg} / \mathrm{m}^{2}$ apresentavam obesidade abdominal, para ambos os sexos e gerações.

\section{DIS C U S S Ã O}

A proposta deste estudo foi descrever a prevalência de sobrepeso, obesidade e obesidade abdominal em nipo-brasileiros. Os resultados mostraram, elevada porcentagem de indivíduos com sobrepeso nesta população em ambos os sexos, sendo $26,1 \%$ e $44,8 \%$ entre os homens de primeira e segunda geração, e 27,9\% e 32,4\% entre as mulheres de primeira e segunda geração, respectivamente. Verificou-se que a segunda geração apresentou prevalências elevadas de sobrepeso, obesidade e obesidade abdominal, quando comparada com a primeira geração, para ambos os sexos.

Entre os indivíduos de ambos os sexos da primeira geração, as maiores prevalências de sobrepeso e obesidade foram a partir dos 65 anos de idade. Na segunda geração, estas prevalências foram maiores entre os indivíduos abaixo de 45 anos até 69 anos

Estudos apontam que as prevalências de sobrepeso e de obesidade, relativamente maiores em populações migrantes, podem ser reflexas do

Tabela 3. Prevalência de sobrepeso, obesidade e obesidade abdominal (OA) em nipo-brasileiros, segundo sexo e geração. Bauru (SP), 2000

\begin{tabular}{|c|c|c|c|c|c|c|c|c|c|c|c|c|}
\hline \multirow{3}{*}{ Homens } & \multicolumn{4}{|c|}{$I M C<25,0 \mathrm{~kg} / \mathrm{m}^{2} \quad(n=301)$} & \multicolumn{4}{|c|}{$\mathrm{IMC}<25-29,9 \mathrm{~kg} / \mathrm{m}^{2} \quad(\mathrm{n}=250)$} & \multicolumn{4}{|c|}{$\mathrm{IMC} \geq 30,0 \mathrm{~kg} / \mathrm{m}^{2} \quad(\mathrm{n}=63)$} \\
\hline & \multicolumn{2}{|c|}{ Sem OA } & \multicolumn{2}{|c|}{ Com OA } & \multicolumn{2}{|c|}{ Sem OA } & \multicolumn{2}{|c|}{ Com OA } & \multicolumn{2}{|c|}{ Sem OA } & \multicolumn{2}{|c|}{ Com OA } \\
\hline & $\mathrm{n}$ & $\%$ & $\mathrm{n}$ & $\%$ & $\mathrm{n}$ & $\%$ & $\mathrm{n}$ & $\%$ & $\mathrm{n}$ & $\%$ & $\mathrm{n}$ & $\%$ \\
\hline Primeira geração & 85 & 90,4 & 9 & 9,6 & 6 & 7,1 & 29 & 82,9 & - & - & 5 & 100,0 \\
\hline \multirow{2}{*}{ Segunda geração } & 191 & 92,3 & 16 & 7,7 & 72 & 33,0 & 143 & 66,5 & - & - & 58 & 100,0 \\
\hline & \multicolumn{4}{|c|}{$\chi^{2}=0,29 p=0,59$} & \multicolumn{4}{|c|}{$\chi^{2}=3,75 \quad p=0,06$} & & & & \\
\hline \multirow{3}{*}{ Mulheres } & \multicolumn{4}{|c|}{$\mathrm{IMC}<25,0 \mathrm{~kg} / \mathrm{m}^{2} \quad(\mathrm{n}=422)$} & \multicolumn{4}{|c|}{ IMC25-29,9kg/m² $(n=227)$} & \multicolumn{4}{|c|}{$\mathrm{IMC} \geq 30,0 \mathrm{~kg} / \mathrm{m}^{2} \quad(\mathrm{n}=67)$} \\
\hline & \multicolumn{2}{|c|}{ Sem OA } & \multicolumn{2}{|c|}{ Com OA } & \multicolumn{2}{|c|}{ Sem OA } & \multicolumn{2}{|c|}{ Com OA } & \multicolumn{2}{|c|}{ Sem OA } & \multicolumn{2}{|c|}{ Com OA } \\
\hline & $\mathrm{n}$ & $\%$ & $\mathrm{n}$ & $\%$ & $n$ & $\%$ & $\mathrm{n}$ & $\%$ & $\mathrm{n}$ & $\%$ & $n$ & $\%$ \\
\hline Primeira geração & 59 & 73,8 & 21 & 26,3 & 3 & 8,8 & 31 & 91,2 & - & - & 8 & 100,0 \\
\hline \multirow[t]{2}{*}{ Segunda geração } & 275 & 80,4 & 67 & 19,7 & 31 & 16,3 & 162 & 83,9 & - & - & 59 & 100,0 \\
\hline & \multicolumn{4}{|c|}{$\chi^{2}=1,74 p=0,18$} & \multicolumn{4}{|c|}{$\chi^{2}=1,19 p=0,27$} & & & & \\
\hline
\end{tabular}

Valores: $\chi^{2}$ : qui-quadrado e $p$ : valor de $p$. 
impacto da ocidentalização no estilo de vida e na dieta desses grupos étnicos ao se fixarem em outros países ${ }^{21,22}$.

Franco ${ }^{21}$, a fim de confirmar essa hipótese em um estudo sobre o papel da aculturação em nipo-brasileiros, encontrou maior prevalência de sobrepeso e obesidade nos indivíduos da segunda geração, quando comparados aos de primeira, sendo esses resultados semelhantes aos deste estudo. Uma das possíveis explicações do autor é a influência da aculturação na segunda geração, a qual parece apresentar uma menor resistência à adoção de um novo estilo de vida, em relação à primeira, ser naturalmente mais jovem e não apresentar um comportamento tão conservador em relação aos hábitos alimentares. Além disso, deve-se também destacar que a maior parte desses nipo-brasileiros vive em área urbana (90\% em 1988 vs $45 \%$ em 1958), o que pode diminuir o gasto energético na atividades cotidianas.

Lerário et al. ${ }^{22}$, em um estudo realizado com este mesmo grupo populacional, também encontraram elevada prevalência de sobrepeso nos indivíduos de segunda geração, quando comparadas com a primeira (29,0\% vs $17,0 \%$; $p<0,05)$, apesar de o critério adotado para os sobrepeso ter sido diferente $\left(I M C \geq 26,4 \mathrm{~kg} / \mathrm{m}^{2}\right)$, reforçando, mais uma vez, a hipótese de que o hábito alimentar, associado à pouca atividade física, são fatores responsáveis pela elevada prevalência da sobrepeso nesta população adulta.

Trabalho semelhante foi realizado por Yano et al. ${ }^{23}$, no qual foram estudados os fatores de risco para o DM2 e doenças cardiovasculares em japoneses residentes no Hawai. Verificou-se que, após a incorporação do estilo de vida ocidentalizado, rapidamente adotado pela comunidade nipo-americana, houve um aumento nos fatores de risco para aterosclerose, como dislipidemia, obesidade e DM2, confirmando a influência dos fatores ambientais na etiologia dessas doenças.

Resultados de outro estudo ${ }^{24}$ sobre a influência da ocidentalização no estilo de vida e o aparecimento de doenças da carótida interna, mostram que a taxa de mortalidade por doença cardiovascular em nipo-americanos foi, aproximadamente, 50\% menor, quando comparada aos caucasianos durante a década de 50 . Porém, na década de 70, após um aumento gradual, atingiu, registrou-se valores muito similares aos encontrados entre a população caucasiana. Assim sendo, os autores concluíram que os nipo-americanos quando expostos a fatores de risco, apresentam doenças na carótida interna e, conseqüentemente, um desenvolvimento maior de doenças ateroscleróticas ao redor dos 20 anos de idade, ou seja, muito mais jovens quando comparados aos japoneses residentes no Japão.

Watanabe et al. ${ }^{24}$ citam a que as diferenças nos hábitos alimentares existentes entre os nipo-americanos e os japoneses nativos, não são em relação à quantidade de energia consumida, mas sim devido à maior contribuição das gorduras no valor energético total ingerido.

Esses dados podem ser confirmados por outros estudos com nipo-brasileiros que revelaram um elevado consumo de óleos e gorduras pelos indivíduos da primeira geração, quando comparados aos de segunda geração ${ }^{25}$. Quando este fato encontra-se associado a uma diminuição no grau de atividade física, ambos podem ser os principais responsáveis pelos altos valores de IMC e CC na segunda geração.

Por fim, apesar da impossibilidade de comparação direta com os resultados de trabalhos realizados com nipo-americanos ${ }^{12,23,24}$, e com residentes no Japão ${ }^{9,11,24}$, devido às diferenças de estrutura etária, as taxas de obesidade observadas neste grupo populacional $(3,7 \%$ e $12,0 \%$ nos homens, e 6,6\% e 9,9\% nas mulheres, respectivamente na primeira e segunda geração), são diferentes das taxas observadas na população residente no Japão $(2,0 \%-3,0 \%)^{8,11}$, porém não se sabe ao certo o motivo destas diferenças. Contudo, cabe ressaltar que o Brasil vem apresentando um aumento nas taxas de sobrepeso e obesidade, especialmente nos homens. As últimas pesquisas de orçamento familiar, realizadas nas 3 últimas décadas, mostram uma projeção claramente epidêmica da obesidade, especialmente no sexo 
masculino e na população de menor renda familiar $^{26}$. O desenho transversal do presente estudo não permite estabelecer relações de causa e efeito. Deve-se considerar a possibilidade da presença de viés nos resultados encontrados, devido à não participação de alguns indivíduos. Entretanto acredita-se que, caso os mesmos fossem incluídos, estes resultados não sofreriam grandes mudanças.

Os resultados deste estudo mostraram que os nipo-brasileiros apresentam elevadas prevalências de sobrepeso e obesidade abdominal, sendo necessárias estratégias efetivas que devem ser desenvolvidas e aplicadas para reverter este quadro, a fim de prevenir doenças crônicas futuras e impedir o agravamento das condições de saúde desta população.

\section{REFERÊ N CIAS}

1. National Center for Health Statistics. Plan and operation of the Third National Health and Nutrition Examination Survey, 1988-1994. Vital Statistics. 1994; 32(1):1-105.

2. Flegal KM, Carroll RJ, Kuczmarski RJ, Johnson CL. Overweight and obesity in the United States: prevalence and trends, 1960-1994. Int J Obes Relat Metab Disord. 1998; 22(1):39-47.

3. World Health Organization. Obesity: preventing and managing the global epidemic. Geneva; 1997. WHO -Technical Report Series, 894.

4. Must A, Spadano J, Caokley EH, Field AE, Colditz $\mathrm{G}$, Dietz WH. The disease burden associated with overweight and obesity. JAMA. 1999; 282(16): 1523-9.

5. Shiwaku K, Annurad E, Enkhmaa B, Nogi A, Shimono K, Yamane Y, et al. Overweight Japanese with body mass indexes of 23.0-24.9 have higher risks for obesity-associated disorders: a comparison of Japanese and Mongolians. Int J Obes. 2004; 28(1):152-8.

6. Annurad, E, Shiwaku K, Nogi A, Kitajima K, Enkhmaa B, Shimono K, et al. The new BMl criteria for Asians by the Regional Office for the Western Pacific Region of WHO are suitable for screening fo overweight to prevent metabolic syndrome in elder japanese workers. J Occup Health. 2003; 45(6):335-43.

7. Popkin BM, Daok CM. The obesity epidemic is a worldwide phenomenon. Nutr Rev. 1998; 56(6):106-14.
8. Health Promotion and Nutrition Research Association: The National Nutrition Survey in Japan, 2000. Ministry of Health, Labor and Welfare, Japan. Tokyo: Daiichi Publishing Co; 2002 (in Japanese).

9. Yoskiike N, Kaneda F, Takimoto H. Epidemiology and obesity and public health strategies for its control in Japan. Asia Pacific J Clin Nutr. 2002; 11(Suppl):S727-31.

10. Souza RKT, Gotlieb SLD. Mortality among Japanese migrants living in state of Paraná, Brasil. Rev Saúde Pública. 1999; 33(3):262-72.

11. Yoshiike N, Seino F, Tajima S, Arai Y, Kawano M, Furuhata $T$, et al. Twenty-year changes in the prevalence of overweight in Japanese adults: the National Nutrition Survey 1976-1995. Obes Rev. 2002; 3(3):183-90.

12. Fujimoto WY, Bergstrom RW, Boyko EJ, Kinyon JL, Leonetti DL, Newell-Morris LL, et al. Diabetes and diabetes risk factors in second and third generation Japanese-Americans in Seattle, Washington. Diab Res Clin Pract. 1994; 24(1):S43-52.

13. Hara H, Egusa G, Yamakido M, Kawate R. The high prevalence of diabetes mellitus and hyperinsulinemia among the Japanese-Americans living in Hawaii and Los Angeles. Diab Res Clin Pract. 1994; 24(1):S37-42.

14. Egusa G, Yamane K. Lifestyle, serum lipids and coronary artery disease: comparison of Japan with the United States 2004; 11(6):334-12.

15. Ferreira SRG, Franco LJ, Gimeno SGA, lochida LC, lunes $\mathrm{M}$. Is insulin or its precursor independently associated with hypertension? An Epidemiological Study Japanese-Brazilians. Hypertension. 1997; 30(3 Pt 2):641-5.

16. Gimeno SG, Ferreira SR, Franco LJ, Hirai AT, Matsumura L, Moises RS. Prevalence and 7-year incidence of type 2 diabetes mellitus in a JapaneseBrazilian population: an alarming public health problem. Diabetologia. 2002; 45(2):1635-8.

17. Ferreira SRG, lunes $M$, Franco $L$, lochida LC, Hirai A, Vivolo MA, et al. Disturbances of glucose and lipid metabolism in first and second generation Japanese-Brazilians. Diab Res Clin Pract. 1996; 34(Suppl):S59-S63.

18. World Health Organization. Physical status: the use and interpretation of athropometry. Geneva; 1995. WHO -Technical Report Series, 854.

19. Inoue S, Zimmet P, Caterson I, Chunming C, Ikeda Y, Khalid AK. WHO Western Pacific Region - The Asia - Pacific perspective: Redefining obesity and its treatment. Melbourne: Health Communication Australia; 2000. 
20. Stata statistical software: release 7.0. Lakeway Drive, (TX): College Station.

21. Franco LJ. Diabetes in Japanese-Brazilians: influence of the acculturation process. Diab Res Clin Pract. 1996; 34(Suppl):S51-7.

22. Lerario DDG, Gimeno SGA, Franco LJ, lunes M, Ferreira SRG. Diabetes na comunidade nipo-brasileira. Rev Saúde Pública. 2002; 36(1): 4-11.

23. Yano K, MacLean CJ, Reed DM, Shimizu Y, Sasaki $\mathrm{H}$, Kodama K, et al. A comparison of the 12-year mortality and predictive factors of coronary heart disease among Japanese men in Japan and Hawaii. Am J Epidemiol. 1988; 127(3):476-87.

24. Watanabe H, Yamane K, Egusa G, Kohno N. Influence of westernization of lifestyle on the progression of IMT in Japanese. J Atheroscler Throm. 2004; 11(6):330-4.

25. Freire RD, Cardoso MA, Shinzato AR, Ferreira SRG, Japanese Brazilian Diabetes Study Group. Nutritional status of Japanese-Brazilians subjects: comaprison across gender and generation. $\mathrm{Br} \mathrm{J}$ Nutr. 2003; 89(5):705-12.

26. Instituto Brasileiro de Geografia e Estatística. Análise da disponibilidade domiciliar de alimentos e do estado nutricional no Brasil. Pesquisa de Orçamentos Familiar (POF) 2002/2003. Rio de Janeiro; 2004.

Recebido em: 5/12/2005

Versãoi final reapresentada em: 16/1/2008 Aprovado em: 18/3/2008 